
géohistorique et enjeux géopolitiques

\title{
L'Iran au travers du prisme géopolitique
}

\section{Pierre Pahlavi}

\section{(2) OpenEdition Journals}

Édition électronique

URL : https://journals.openedition.org/geohist/1487

DOI : $10.4000 /$ geohist. 1487

ISSN : 2264-2617

Éditeur

Association française de la Revue de géographie historique

\section{Référence électronique}

Pierre Pahlavi, «L'Iran au travers du prisme géopolitique », Revue de géographie historique [En ligne], 12 | 2018, mis en ligne le 20 mai 2018, consulté le 12 juin 2021. URL : http://journals.openedition.org/ geohist/1487 ; DOI : https://doi.org/10.4000/geohist.1487

Ce document a été généré automatiquement le 12 juin 2021.

\section{(c) (†) $\ominus$}

Ce(tte) œuvre est mise à disposition selon les termes de la Licence Creative Commons Attribution -

Pas d'Utilisation Commerciale - Pas de Modification 4.0 International. 


\title{
L'Iran au travers du prisme géopolitique
}

\author{
Pierre Pahlavi
}

\section{Introduction}

1 Plusieurs facteurs géographiques confèrent à l'Iran une solide réputation de pivot géopolitique. La géologie a fait de ce plateau montagneux et partiellement désertique une forteresse naturelle ceinturée de montagnes qui dominent les plaines de la Mésopotamie, de l'Indus et de la mer Caspienne. L'isthme médique, pris en tenaille entre la plaque Eurasie (Caucase et plaine du Touran), la péninsule arabique et le souscontinent indien, cadenasse l'ensemble régional. Au fil des siècles, les hommes ont bâti sur ce socle un carrefour, politique, économique et culturel, situé à l'intersection des mondes arabe, turco-caucasien et indo-européen.

2 L'Iran se classe non seulement parmi les vingt plus grands pays du globe mais il jouit également d'une position stratégique clé puisque, avec l'émiettement des empires qui le côtoyaient jadis, son territoire se retrouve entouré d'une quinzaine d'États limitrophes. À l'est se trouvent l'Afghanistan et le Pakistan. Au nord, le Caucase avec l'Arménie, l'Azerbaïdjan ; l'Asie Centrale avec le Turkménistan, et 740 km de côtes sur la mer Caspienne. Les frontières occidentales sont partagées avec la Turquie et l'Irak, finissant au Chatt-el-Arab ${ }^{1}$. Au Sud, le Golfe persique et le Golfe d'Oman forment l'intégralité de sa limite méridionale. Tous les éléments de cette situation singulière se conjuguent pour faire du « pays des Aryens $»^{2}$ un verrou stratégique et une plaque tournante du grand système eurasiatique. 
PHYSIOGRAPHY OF IRAN

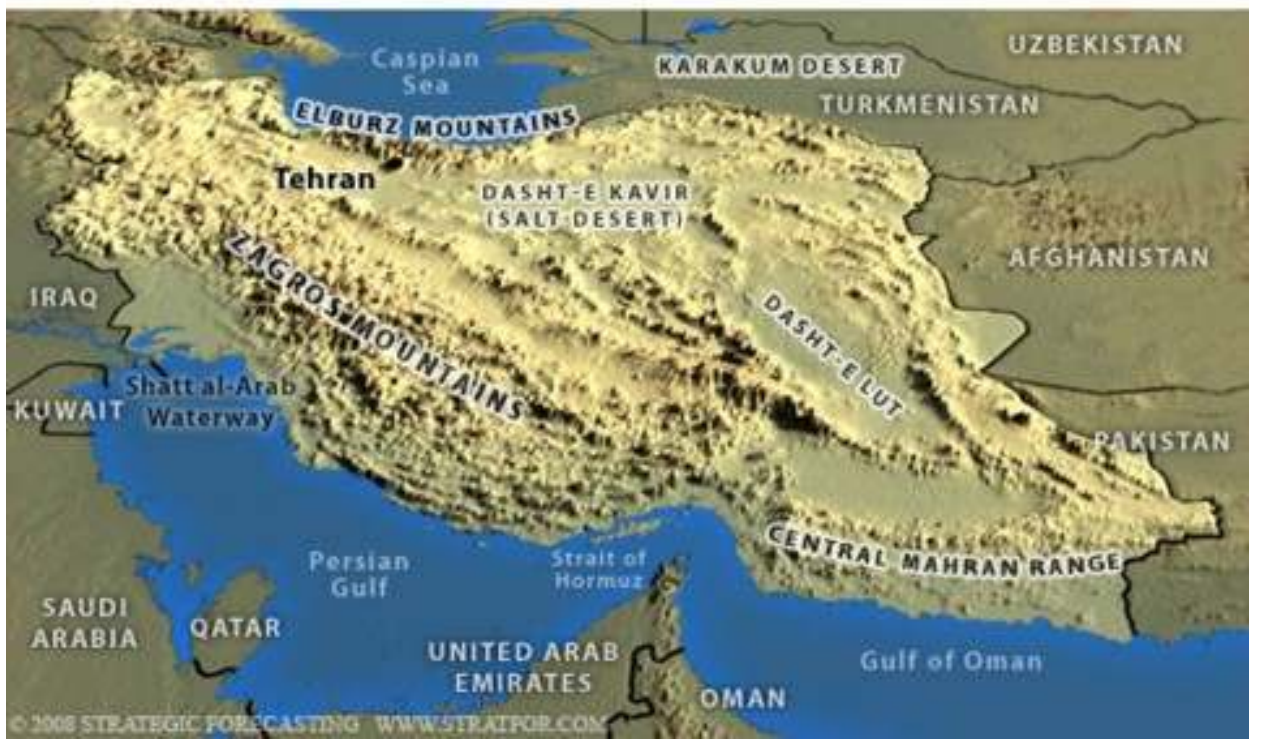

Source: Worldview

3 L'ère des hydrocarbures et la découverte de formidables gisements de gaz et de pétrole ont parachevé ce statut de pivot. Avec les quatrièmes réserves prouvées d'or noir au monde et les premières réserves prouvées de gaz naturel, l'Iran est, depuis plus d'un siècle, l'objet de toutes les convoitises ; une manne qui est appelée à demeurer " un facteur déterminant de son histoire tout au long du XXI ${ }^{e}$ siècle » (Djalili, 23). A ses richesses énergétiques s'ajoute le contrôle stratégique que Téhéran est en mesure d'exercer sur le détroit d'Ormuz à travers lequel transite 30\% du commerce mondial d'hydrocarbures.

4 Pays charnière, verrou stratégique, carrefour de communication, nœud de pèlerinage et d'échanges commerciaux, l'entité iranienne est perçue comme un rouage essentiel entre les grandes plaques tectoniques du système international. Pour autant, la question se pose de savoir dans quelle mesure le destin de l'Iran est « déterminé » par cette situation géostratégique. Peut-on dire à la manière de Spykman que la géographie est « le facteur le plus fondamental » pour l'Iran «car il est le plus permanent » (Spykman, 41) ou, plus directement encore, à la manière de Napoléon, que son devenir « est dans la géographie » (Challiand, v) ? Certes, non. L'Iran, pas plus qu'un autre pays, ne peut être réduit à sa position sur la mappemonde : "c'est la géographie qui a fait de l'Iran un élément incontournable du Grand Moyen-Orient et de l'Asie, mais, comme le note Kaplan, elle ne peut décider de ce que l'Iran fera de sa position favorable car cela ne relève que des décisions humaines » (Kaplan, 280-81).

5 En marge de la géopolitique classique qui se limite à l'impact de la géographie physique, une voie alternative consiste à examiner la manière dont cette réalité permanente et objective est prolongée, reconstruite et réinterprétée historiquement sous la forme d'un prisme subjectif qui détermine à son tour la perception que les états ont les uns des autres et de leur rôle respectif dans le jeu international (Dodds). Ainsi, sur fond du décor géographique qui fixe la structure de ce jeu, se développe un scénario articulé autour d'une distribution des rôles et des fonctions attribuées à chacun. Le complexe de sécurité Moyen-Orient n'est pas exempt de ces schémas durables d'amitié 
et d'antagonisme (amity and enmity patterns) qui, au moins autant que la donnée permanente de la géographie, influencent les interactions entre acteurs régionaux et, in fine, la logique du jeu collectif (Buzan et Waever, 45-55) ${ }^{3}$.

6 L'objectif de cet article est de démontrer que, en plus d'être influencé par les paramètres tangibles d'ordre géostratégique, l'Iran et son destin sont très largement déterminés par la contradiction profonde qui existe entre les "fonctions » géopolitiques que lui attribuent les autres états, régionaux et extrarégionaux, et le rôle que ce pays estime devoir être le sien sur l'échiquier moyen-oriental et international. La mise en contraste de ces visions géopolitiques - souvent divergentes - fait apparaître la complexité de la relation entre l'Iran et ses partenaires internationaux ainsi que le nombre limité des options stratégiques qui s'offre à ce pays afin de poursuivre ses ambitions et objectifs stratégiques.

\section{Le regard des voisins eurasiatiques}

7 En règle générale, les autres états cantonnent l'Iran à un rôle de moyen au service de leurs intérêts stratégiques. Une tendance soulignée par l'Iranologue Shireen Hunter lorsqu'elle écrit que : « aux yeux des principaux acteurs internationaux, l'Iran n'a pas de valeur qui lui est propre: il n'est utile que pour atteindre d'autres objectifs comme servir d'État tampon mais non pas comme un acteur à part entière auquel serait reconnu une responsabilité souveraine » (Hunter). Commençons par ses voisins eurasiatiques.

\section{A. Les perspectives turcique et chinoise}

8 Vue sur un axe Est-Ouest, la Perse a traditionnellement été considérée comme un corridor naturel d'invasion. Venant de l'est, le Touran submerge le Khwarezm à plusieurs reprises avec les conquêtes des Gengis Khan, Hulagu Khan et autres Tamerlan. Aux yeux de ces envahisseurs centre-asiatiques, la « fonction " géopolitique de l'Iran du Moyen Âge se réduit largement à celle d'une " province à prendre » en vue de l'édification des grands empires eurasiatiques qui s'échelonne entre le XIIIe et le XVe siècle. Condamnée à subir ces invasions mongoles, l'Iran doit attendre l'avènement des Safavides, première dynastie indigène en près de 1000 ans, pour recouvrer un peu de sa souveraineté.

9 A peine s'estompe-t-elle à l'est que la pression turcique, dans un mouvement de reflux, s'exerce à partir du Ponant sous la forme de l'expansionnisme ottoman. Après les invasions seldjoukides, cet impérialisme, moins violent mais plus durable culmine, entre le XVe et le XIXe siècle par une forte concurrence entre la Sublime porte et la dynastie Safavide pour le contrôle du monde musulman. Une compétition régionale d'autant plus féroce qu'elle se teinte d'une rivalité religieuse entre les Califes turcs, devenus champions d'un sunnisme hégémonique, et les Chahs qui épousent la cause d'un chiisme résistant. Tout au long de leur règne, les souverains safavides, Afsharides et Zands sont aux prises avec ce péril permanent.

$10 \mathrm{Si}$, depuis la mutation des empires en États-nations, Turcs et Iraniens ont enterré la hache de guerre, c'est plus par affaiblissement mutuel que par manque d'ambition (Elik). Figé au cours de la Guerre froide, la rivalité turco-iranienne ré-émerge depuis le Printemps arabe et la recomposition du complexe de sécurité moyen-oriental. Ankara ne cache plus ses ambitions néo-ottomanes - le vizir Ahmet Davutoğlu ayant été jusqu'à 
déclarer : « le Moyen-Orient nous appartient»(Daloglu) - et les traités qui, de celui de Qasr-e Chirin (1639) à celui de Lausanne (1923), avaient permis d'apaiser les litiges territoriaux, sont de plus en plus couramment remis en cause de part et d'autre (AlAwsat). La Turquie d'Erdoğan est certes le voisin le moins « envahissant » de l'Iran mais il n'en demeure pas moins un acteur ambitieux pour qui la République chiite reste un obstacle géopolitique à l'extension de son influence dans le monde turco-arabe et turco-eurasiatique.

Document 2 : La nouvelle route de la Soie de la Chine et l'Iran

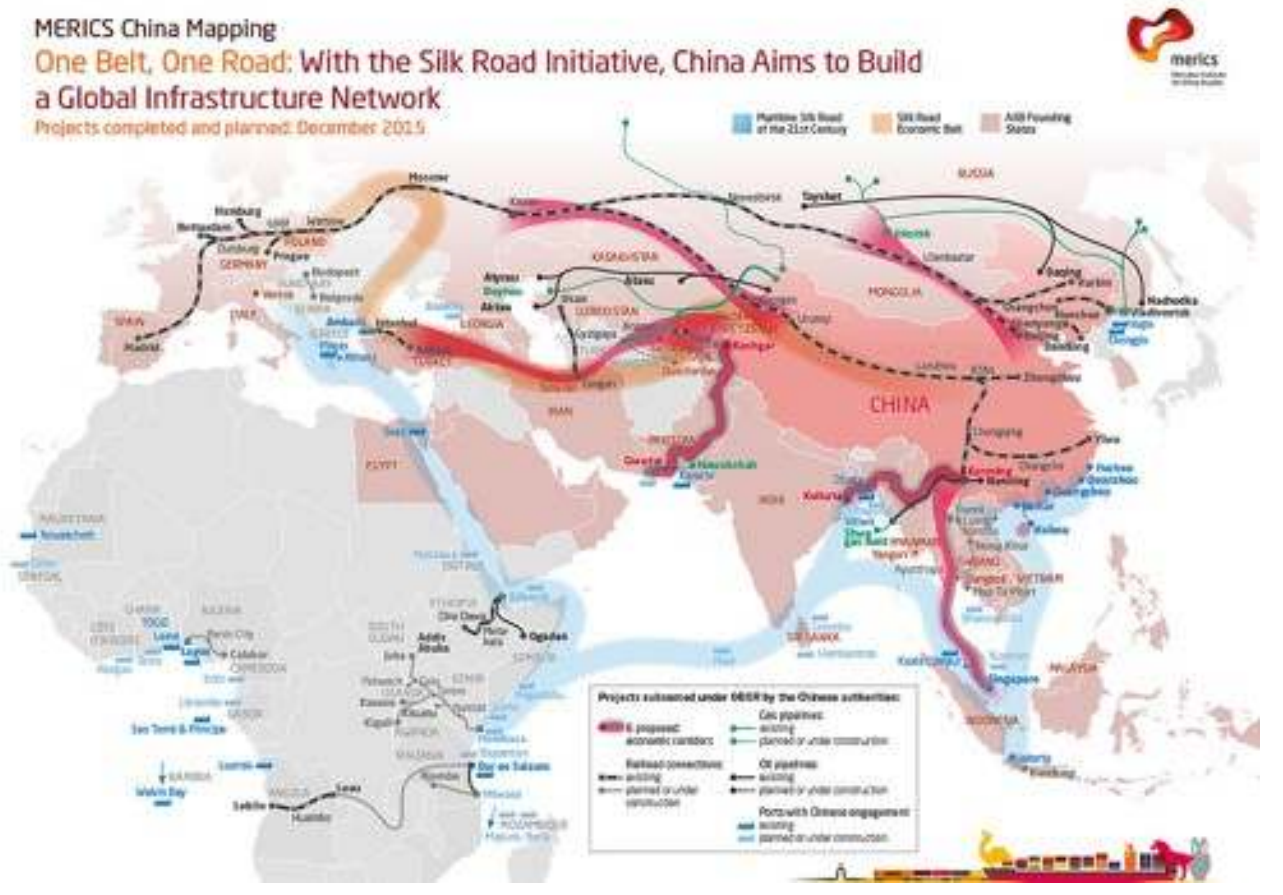

Source : Eurasia News Online

Pratiquant un impérialisme plus économique que militaire, la Chine a plutôt coutume de voir en l'Iran un tronçon nécessaire à l'édification de sa fameuse Route de la Soie et, de manière plus indirecte, une plate-forme pour l'extension de son influence eurasiatique. Les stratèges chinois ne se trompent pas sur le rôle charnière que doit jouer l'Iran dans leur projet high-tech de "One Belt, One Road »: «De nombreux pays sont importants dans ce projet », déclare l'un d'entre eux, "mais l'Iran est au centre de tout" (Erdbrink). Semblables aux fibres d'une écharpe géopolitique, chemins de fer, voies maritimes, gazoducs et oléoducs enserrent le maillon iranien dans un vaste réseau eurasiatique patiemment tissé par les dirigeants chinois (Blanchard).

Le développement des liens de communication et d'échange avec l'Iran obéit à la promotion d'intérêts stratégiques de l'Empire du Milieu. En plus de trouver dans l'Iran un débouché pour ses exportations manufacturières, la RPC y voit une source d'approvisionnement pour ses besoins considérables en matière de pétrole et gaz naturel (Ilminskaya). En outre, le développement des liens sino-iraniens permet aux Chinois de gagner un accès direct au Moyen-Orient : "Pékin voit l'Iran comme un moyen de limiter l'influence U.S. au Moyen-Orient » (Harold et Nader). Dans le scénario chinois comme dans ceux écrits ailleurs, Iran se voit donc prescrire d'interpréter un rôle au service des ambitions et stratégies d'influence d'autres puissances. 


\section{B. La perspective arabe}

13 A partir du Sud, du Levant ou de la péninsule arabique, l'Iran se présente comme un verrou géo-culturel fermant le passage vers le Caucase et à l'Asie centrale. Sur le constat géographique se greffe une profonde et ancienne hostilité qui, à elle seule, illustre le concept des amity and enmity patterns et la notion de " rôles géopolitiques " (Shayan, 26).

14 L'antagonisme irano-arabe prend la forme d'une relation dominant-dominé qui débute dès 642 , lorsque les hommes des sables écrasent l'empire rupestre des sassanides à Nahāvand et que les Califes arabes remplacent le zoroastrisme des Iraniens par l'Islam et la langue persane par celle du Prophète (Frye, 46). Sous le règne des Omeyades, les Persans convertis, appelés mawlas (musulmans non-arabes), sont soumis à de multiples humiliations et écartés des postes de responsabilité (Clawson, 16-18). D'emblée, l'imposition de la langue arabe et celle de l'Islam se heurtent au fort sentiment d'exceptionnalisme des Iraniens qui rejettent la première et transforment la seconde en lui donnant une forme gallicane. Religion des faibles et des opprimés obéissant aux dogmes du moqavemat [résistance] et du zolm [tyrannie/injustice], le Chiisme iranien est par ailleurs truffé de techniques de mystification intellectuelle développées par le clergé iranien qui, comme les doctrines du taqiya, du ketman ou du khodeh, visent à assurer la survie et l'épanouissement de la cause chiite face à la domination sunnite.

Dès le Moyen Age, la relation du suzerain arabe avec ses vassaux persans a été marquée par le sceau de la méfiance mutuelle. Dans une fameuse lettre adressée au gouverneur d'Irak, Muawiyah, le premier Calife omeyyade, écrit: « Soyez vigilants envers les Iraniens et ne les traitez jamais comme des égaux des Arabes ». Une méfiance attisée par l'iranisation du dogme musulman et l'ambivalence prêtée à ses disciples. Caractérisée par une dualité inhérente, le chiisme iranien, naviguant sans cesse entre l'explicite et l'implicite, le révélé et l'indicible, a, très tôt, poussé les Arabes à regarder les " hérétiques perses " avec la plus grande suspicion. Pas étonnant d'ailleurs qu'entre autres termes péjoratifs utilisés pour qualifier les Iraniens, les Arabes se réfèrent encore souvent aujourd'hui à eux comme des «ajam» signifiant " ambiguës et indéchiffrables » par opposition à « arabi » c'est-à-dire « clairs et limpides » (Bosworth, 700-1).

Qu'ils soient authentiques ou volontairement amplifiés (Adib-Moghaddam), les préjugés continuent d'influencer les relations irano-arabes qui, depuis un siècle, oscillent entre des périodes de coexistence froide et de conflit larvé voir ouvert (1980-1988): à la haine des uns (al-Zahed) répond toujours l'«iranophobie» des autres (Shariatinia). En rouvrant les lignes de fracture confessionnelle entre chiites et wahhabites, la révolution islamique de 1979 est venue aggraver le duel idéologique qui sous-tend la rivalité géopolitique. Même si elle donne parfois l'illusion d'une compétition froide et rationnelle, cette rivalité continue de plonger ses racines dans la méfiance séculaire des Arabes à l'égard des héritiers des Achéménides et s'incarne autant dans le Conseil de coopération du golfe (GCC) établi en 1981 pour contenir l'Iran que dans la thèse du "Croissant chiite » qui prête aux « hérétiques perses » la volonté d'imposer leur domination à l'ensemble de la région (Shahandeh et Warnaar).

Avec le Printemps arabe, l'Iran des mollahs apparaît plus que jamais aux yeux des puissances arabes comme une puissance adverse et ambivalente se dressant face à leurs 
ambitions régionales. La constitution progressive de l'axe Téhéran-Bagdad-DamasBeyrouth vient renforcer la thèse du " rôle géopolitique " que les capitales arabes l'accusent d'avoir toujours joué : celui d'un verrou idéologique, politique et donc stratégique faisant obstacle à l'affirmation de leur hégémonie mais aussi celui d'un corps étranger qu'il faudrait dissoudre pour rétablir l'harmonie de l'Umma. Face à ce défi, les puissances arabes se tournent vers les deux approches qu'elles ont toujours adoptées vis-à-vis de l'Iran : l'offensive et, en attendant que celle-ci ne redevienne envisageable, l'endiguement (Mousavian).

\section{La perspective russe}

Pour les Russes, l'Iran a toujours été une passerelle naturelle donnant accès aux mers chaudes. Une vision géopolitique élevée au rang de dogme comme en atteste Le Testament de Pierre Ier exhortant les dirigeants russes à étendre leur domination vers les territoires qui séparent la Russie des mers du Sud : «[il faut] hâter la décadence de la Perse, pénétrer jusqu'au golfe Persique ; rétablir, si c'est possible, par la Syrie, l'ancien commerce du Levant, et avancer jusqu'aux Indes, qui sont l'entrepôt du monde." Apocryphe, ce document n'en trace pas moins l'une des priorités les plus pérennes de la stratégie russe (Gaume, 10).

De Catherine la Grande à Vladimir Poutine, les Tzars ont appliqué ce dogme d'autant plus religieusement qu'il répond à une réalité géopolitique permanente : «Depuis Pierre le Grand, note Spykman, la Russie n'a d'autre obsession que de sortir de son enclavement continental et de s'ouvrir un accès à l'océan " (Sloan, 17). La constance de la géographie et celle de l'axiome stratégique se sont donc conjuguées pour conférer à l'isthme médique le statut de "territoire critique " pour l'expansion de l'influence de la Russie au Moyen-Orient (Kaplan, 104).

$20 \mathrm{Au}$ XIXe siècle, cette obsession s'exprime dans le cadre du Grand jeu d'influence opposant la Russie des Romanov à la couronne britannique pour le contrôle de l'Asie centrale et de la vieille Perse. À coups d'épée, les Tzars taillent dans la chair de l'Iran et se fraient un passage vers les mers chaudes: C'est entre autres le Traité du Golestân (1813), le traité de Turkmanchai (1828) et l'entente russo-britannique de 1907 qui réduisent la souveraineté iranienne et arrachent à la dynastie Qadjar ses territoires caucasiens de Géorgie, d'Iğdır, du Daghestan, du Nakhitchevan, d'Arménie et l'essentiel de l'Azerbaïdjan actuels.

Momentanément freinée par la Révolution bolchevique, la poussée russe vers les mers chaudes prend la forme de l'impérialisme soviétique. Dès 1920, Moscou rétablit son contrôle sur tout le Caucase d'où l'URSS, reprenant à son compte l'œuvre des tsars, continue, tout au long du XXe siècle, à menacer la souveraineté iranienne. Ce seront, entre autres, l'occupation, par l'Armée rouge du nord de l'Iran durant la seconde guerre mondiale (1941); l'encouragement de Staline au rattachement de l'Azerbaïdjan iranien avec son pendant soviétique (1946); mais aussi, pendant la guerre froide, les pressions constantes exercées sur l'empire de Mohammad Reza devenu poste avancé du dispositif U.S. d'endiguement de l'Union soviétique. Parallèlement à l'intervention en Afghanistan, la pression soviétique s'exerce jusqu'à l'écroulement de l'édifice Pahlavi et le triomphe de la révolution islamique avant que, une décennie plus tard, l'« empire éclaté » ne s'écroule à son tour. 


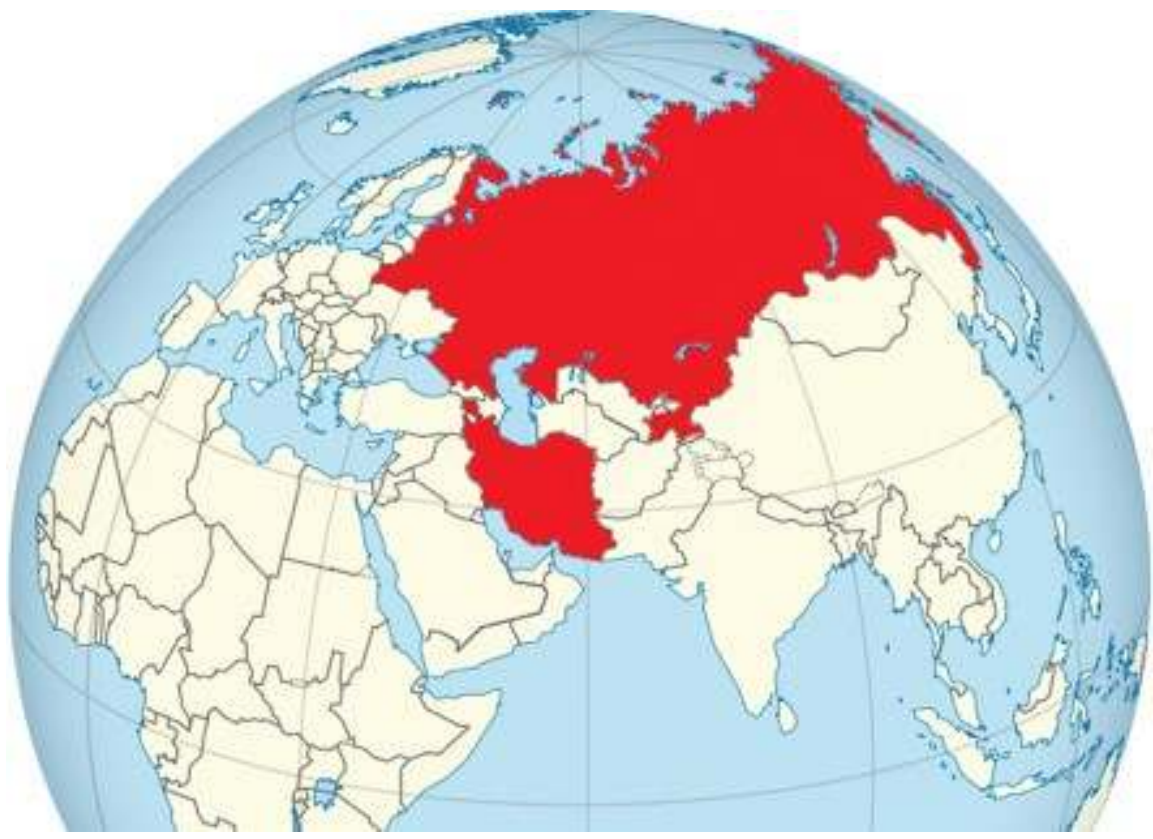

Source : Iran Review

Dans l'équation post-guerre froide de la Russie et dans le schéma mental de ses penseurs stratégiques, l'Iran demeure une variable clé (Shlapentokh, 14-75). Pour les néo-Eurasianistes tels Aleksandr Dugin, la passerelle iranienne est une condition sine qua non à la restauration de la grandeur de la Russie (Dugin, 239-241). Ravivant l'idée d'une grande poussée militaire vers l'ancienne Perse, les nationalistes rêvent de voir les troupes russes « laver leurs pieds dans les eaux chaudes de l'océan Indien » - les « Perses et les Turcs » dussent-ils en faire les frais (Jirinovski, 43-49).

Vladimir Poutine a fait sienne la stratégie d'un grand retour de la Russie au MoyenOrient empruntant la passerelle iranienne. Sans avoir de la sympathie pour les ayatollahs, il sait qu'il a besoin d'eux pour « renverser le déclin géopolitique de son pays » (Tiersky). Dans la promotion d'un ordre multipolaire, le Kremlin n'a jamais considéré l'option iranienne que comme un moyen - un moyen d'autant plus opportun pour percer le monde musulman que le régime islamique est relativement modéré et que son isolement diplomatique ne lui laisse que peu d'autres alternatives. Pour utiliser une image, le tapis persan n'est "qu'une pièce parmi d'autres dans le patchwork de relations stratégiques » (Geranmayeh et Liik) que Moscou rapièce résolument depuis le début du XXIe siècle.

\section{Le regard des puissances extra-régionales}

À l'instar des puissances eurasiatiques, les puissances extrarégionales perçoivent elles aussi l'Iran comme un moyen au service de leurs objectifs stratégiques. Mais, au lieu de le considérer selon un axe Nord-Sud/Est-Ouest et une logique de conquête/passage, ces puissances lointaines regardent le système moyen-oriental à la verticale et de l'extérieur ; une perspective dans laquelle l'Iran apparaît comme un point de chute, une case de l'échiquier eurasiatique qu'il faut contrôler $\mathrm{d}^{\prime}$ " en-haut » pour rayonner sur l'ensemble du jeu. 

l'Iran au XIXe siècle est codifié et systématisé par A. Mahan et H. Mackinder, puis N. Spykman et enfin H. Kissinger et Z. Brzeziński. Ces théoriciens convertis en conseillers stratégiques contribuent à écrire le scénario de la politique moyen-orientale des ÉtatsUnis. En cantonnant l'Iran dans un rôle de "debatable ground", ils scellent, dans une très large mesure, le destin géopolitique de ce pays et démontrent l'influence déterminante que peut avoir la construction de ces "partitions " théoriques sur la réalité stratégique.

\section{A. La vision des pères fondateurs}

Premier à définir le concept de Moyen-Orient dans un article intitulé «The Persian Gulf and International Relations » (1902), l'amiral Alfred Mahan y invite la Grande-Bretagne à contenir la poussée russe en établissant des bases militaires dans le Golfe et notamment en Perse (Gueynard, 17). À peine cinq ans plus tard, Londres signe avec Saint-Pétersbourg un traité partageant l'Iran en deux zones d'influence sans qu'aucun Iranien n'ait été convié aux négociations ou même informé de leur tenue (Kinzer, 2010, 7). En constatant que l'accès aux mers chaudes est pour la Russie un enjeu stratégique majeur, Mahan élabore l'une des bases fondamentales de la stratégie moyen-orientale et iranienne des puissances anglo-saxonnes : l'Iran est une « case critique » qu'il faut à tout prix contrôler pour contrecarrer les ambitions russes. À noter que, loin de se limiter à la sphère théorique, Mahan influence directement des responsables politiques comme Benjamin Tracy, Henry Cabot Lodge ou Theodore Roosevelt et, à travers eux, l'axe de la politique orientale des États-Unis pour plusieurs générations.

Plus influent que Mahan et encore plus obnubilé que lui par le péril russe, l'homme politique et géographe H. J. Mackinder publie un article intitulé " The Geographical Pivot of History » (1904) dans lequel il présente une division du globe entre, d'une part, les «îles périphériques» essentiellement dominées par les puissances maritimes britanniques et nord-américaines et, d'autre part, le World-Island dont le noyau terrestre, appelé Heartland, correspond essentiellement à l'espace occupé par l'Empire russe. Sa thèse est la suivante : "Qui contrôle l'Heartland contrôle l'île Monde ; Qui contrôle l'île Monde contrôle le monde " (Mackinder, 421-437). De cette vision globale, Mackinder tire la prescription suivante : les puissances maritimes doivent tout mettre en œuvre pour empêcher qu'une puissance quelle qu'elle soit puisse un jour dominer le grand bloc continental constitué par l'Eurasie. Pour lui, le meilleur moyen d'hypothéquer les chances de la Russie de s'emparer du Heartland est de développer le contrôle de ce qu'il appelle le «Iranian upland » - bande de terre s'étalant du Kurdistan iranien à la mer de Chine. Une nouvelle fois l'Iran, conçu comme l'élément pivot du « pivot » du monde, est placé au cœur de l'équation géopolitique. 


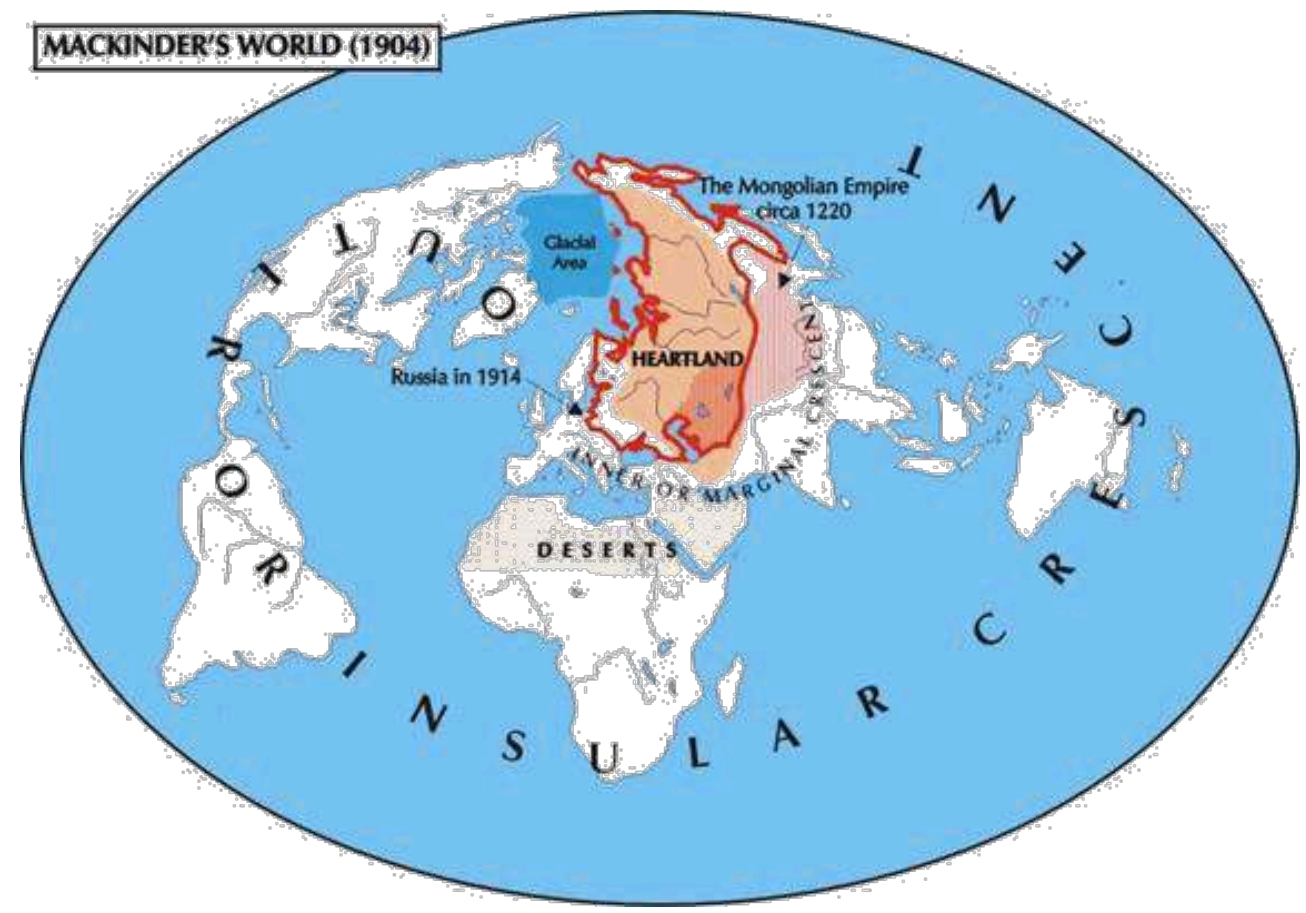

Source : ArchivesEroe

Sans doute n'y a-t-il pas meilleure illustration des théories de Mahan et de Mackinder (et de leurs impacts pour l'Iran) que les circonstances qui ont accompagné l'avènement et la chute Reza Chah (19251941). Au début des années 1920, l'Angleterre de Lord Curzon déroule un cordon sanitaire de la Baltique à l'Asie centrale pour contenir la menace renouvelée qu'exerce la Russie bolchevique, et se met en quête de quelques Porteurs de Sabre pour monter la garde sur les pourtours du Heartland de Mackinder. Du Nord au Sud et d'Ouest en Est ce seront: Mannerheim en Finlande, Pilsudski en Pologne, Antonescu en Roumanie, Moustapha Kemal en Turquie, Tchang Kaï-Chek en Chine et ... Réza Khan en Perse. Deux décennies plus tard, les mêmes considérations géopolitiques entraînent la fin de son règne. Au début de la seconde guerre mondiale, convaincus que l'issue de la guerre dépendra du contrôle du couloir persan, renommé " pont de la victoire ", les alliés décident de se débarrasser de Reza chah soupçonné de ne plus remplir adéquatement sa mission géopolitique et d'entretenir des relations ambiguës avec les puissances de l'axe. À F. D. Roosevelt l'interrogeant sur ce qu'il est advenu du roitelet oriental, Churchill répond en 1941 : «Empire donné, Empire repris » (Kapuscinski, 38).

\section{B. Spykman et le Rimland}

Alors que la seconde guerre mondiale s'achève sur une victoire des alliés, Nicholas $\mathrm{J}$. Spykman adapte les théories de Mahan et de Mackinder à la réalité de ce qui s'apparente déjà à la Guerre froide. À la division mackinderienne il ajoute un raffinement: entre la "périphérie maritime " des puissances anglo-saxonnes et le Heartland - qui désormais correspond aux contours de l'URSS -, Spykman entrevoit un long cordon sanitaire qu'il appelle Rimland. Dans sa conception, ce n'est plus le Heartland mais cette longue ceinture qui devient la clé de la géopolitique mondiale : 
"Qui contrôle le Rimland gouverne l'Eurasie ; qui gouverne l'Eurasie contrôle les destinées du Monde ». En précisant les notions de « Inner or Marginal Crescent » et de «Iranian upland » ébauchées par Mackinder, Spykman met l'accent sur la nécessité d'établir un contrôle sur les pays de cet immense glacis protecteur. De ce dispositif destiné à endiguer et, si possible, étouffer le géant soviétique, l'Iran, avec ses richesses pétrolières et le contrôle qu'il peut exercer sur les voies de communication énergétique, doit devenir l'une des pièces maîtresses - au prix s'il le faut d'une partie de sa souveraineté.

Document 5 : Le Rimland de Spykman (1943) et l'Iran

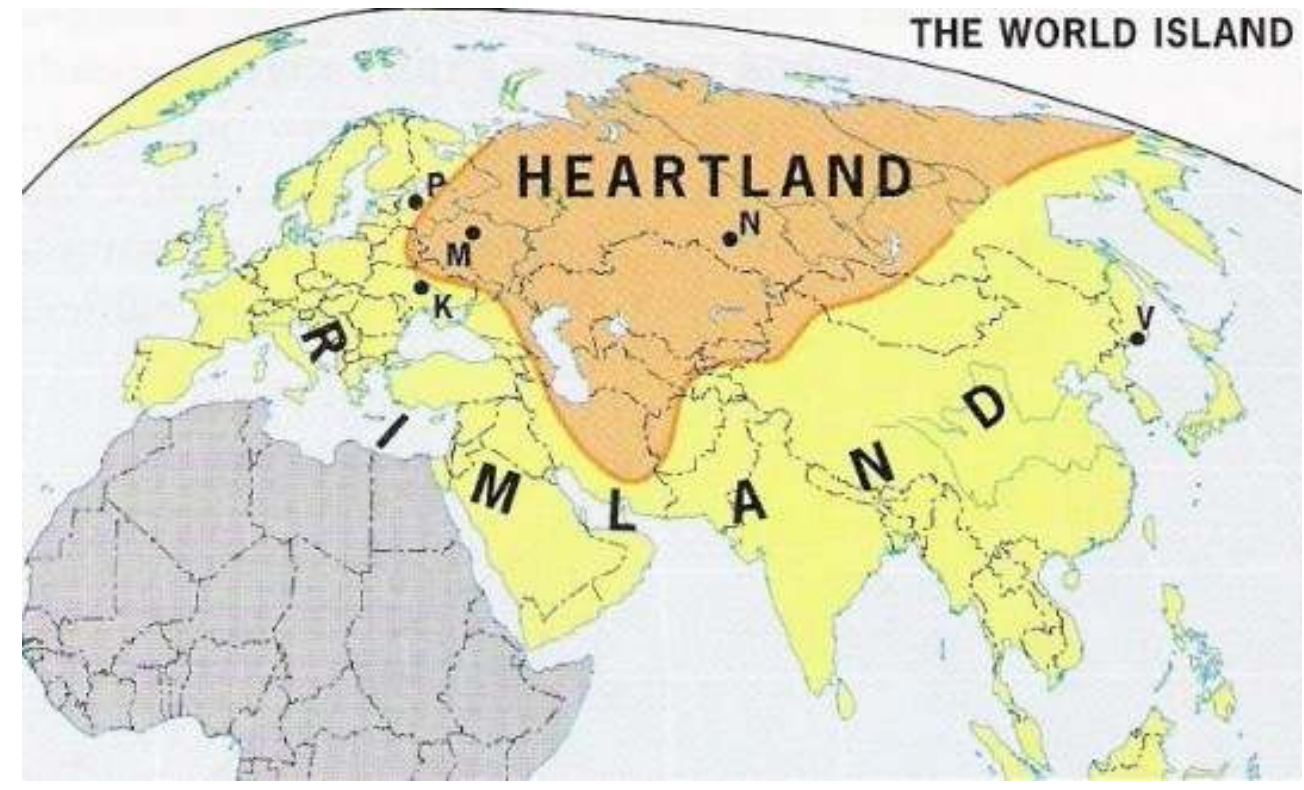

Source : Les yeuxdumonde.fr

Aussitôt formulé, aussitôt appliqué. Dès le lendemain de la seconde guerre mondiale, le «Pont de la Victoire » redevient le « Barrage Perse » et Mohammad Reza est placé sur le Trône du Paon pour remplir, comme son père, le rôle de " garde-barrière » de l'empire soviétique. Le nouveau "protégé » obtient un support sans faille des capitales occidentales qui volent à son secours lorsque son trône vacille face aux ardeurs révolutionnaires du Docteur Mossadegh. C'est l'Opération Ajax d'aout 1953 qui aboutit à une Restauration de la Monarchie iranienne et à une stabilisation du statu quo régional pro-occidental. En retour, les puissances anglo-saxonnes n'ont qu'une exigence : que l'Iran du Chah s'acquitte fidèlement de son rôle de digue anti-soviets. Le maillon perse de la chaîne de défense contre le Communisme est remis au goût du jour par Foster Dulles et la doctrine Eisenhower, adoptée en 1957, renforce son rôle de rempart du "Monde libre». Mohammad Réza, en intégrant le pacte de Bagdad, puis le CENTO, devient, comme son père, l'un des maitres d'œuvre de la stratégie de containment de l'Union soviétique. L'histoire se répète.

\section{Kissinger, Brzeziński et la Ceinture verte}

Pétris des théories de Mahan, Mackinder et Spikman, Henry Kissinger et Zbigniew Brzeziński perpétuent le dogme géopolitique américain tout en lui imprimant un nouveau twist. Au cours des années 1960-1970, ils réalisent que la vielle doctrine 
du containment n'est plus adaptée et que l'Union soviétique de Léonid Brejnev, loin d'être contenu dans le carcan du Rimland, le menace à nouveau de ses tentacules.

Entrevoyant la mutation de la menace soviétique, Henry Kissinger pose un diagnostic sans appel au sujet du régime du chah d'Iran : en plus d'être devenu imprévisible (par son influence au sein de l'OPEP) et incommodant (par ses frasques et sa mégalomanie), il est de moins en moins fiable dans la croisade contre le communisme et il devient urgent de considérer des alternatives: " pour Henry Kissinger, observe Robert D. Kaplan, c'est en agissant suffisamment tôt que l'on évitera de trop lourdes pertes» (Kaplan, 97). Dès 1974, Kissinger envisage un Iran post-monarchique et déclare devant le Conseil national de sécurité: «il faut que le Chah change de politique ou que le Chah s'en aille » (Villemarest, 4). Le géopoliticien sait déjà que, si « la nouvelle doctrine » devra continuer d'être " une application logique des travaux de Mackinder et des raffinements apportés par Spykman ", sa mise en œuvre entraînera nécessairement des bouleversements profonds "voire des conflits » pour les pays du Rimland incluant la Chine, le Pakistan, l'Afghanistan, la Pologne ... mais aussi et surtout l'Iran (Kaplan, 97).

C'est sous l'administration Carter et la férule de son conseiller stratégique, Zbigniew Brzeziński, qu'est identifiée la nouvelle doctrine dite de l'« Arc de crise » impliquant une modification profonde de la partition assignée à l'Iran. Constatant que l'aggravation des lignes de fracture tribales et religieuses peut entraîner la balkanisation du monde musulman et, par voie de conséquence, la déstabilisation du Bloc soviétique, les conseillers stratégiques de la Maison-Blanche, parmi lesquels des islamologues comme Bernard Lewis, préconisent de substituer à la vieille digue fissurée de l'anticommunisme et à ses «Porteurs de Sabre» désuets plantés dans leur tranché du CENTO un nouveau cordon composé d'états fondamentalistes dont la fonction ne serait plus d'endiguer l'URSS mais de l'étouffer. Pour mettre un terme à la sempiternelle " lutte entre l'Ours [russe] et la Baleine [occidentale] » décrite par H. J. Mackinder, il s'agit donc de doter cette dernière d'une nouvelle dentition acérée formée par un chapelet islamiste. Dans le cas de l'Iran, l'espoir est de voir émerger un régime hybride, islamo-laïque sous contrôle de l'armée pro-occidentale et sous supervision de Washington (Pahlavi et Pahlavi, 239-249). Du déroulement de ce que les Iraniens appellent le Karmarband-e Sabz - la ceinture verte - leur pays aura été le premier chantier.

De ce plan de recomposition et de "modernisation " de la vieille enceinte antisoviétique, l'Afghanistan est l'autre laboratoire. Dès le mois d'avril 1978, alors que crépite la révolution iranienne et que Washington a commencé à prendre ses distances avec le régime du Chah, Carter et Brzeziński décident d'apporter l'assistance financière et logistique aux Moudjāhedin afghans laissant se déchaîner sur l'Armée Rouge la guérilla djihadiste. A court terme, le pari est gagnant : c'est le Vietnam de l'Armée rouge. Vingt ans plus tard, Brzeziński s'enorgueillira encore des résultats de ce coup de poker géopolitique : "Qu'est-ce qui est le plus important au regard de l'histoire du monde? Quelques excités islamistes où la chute de l'empire soviétique et la fin de la guerre froide ?» (Brzeziński, 1998) Alors qu'ils se satisfont de voir le piège de l'islamisme se refermer sur l'Union soviétique, il faudra plus de temps aux États-Unis - et les attentats de 2001 à New York - pour comprendre qu'ils venaient d'ouvrir la Boîte de Pandore d'où s'échappera le mauvais génie du djihad international. 


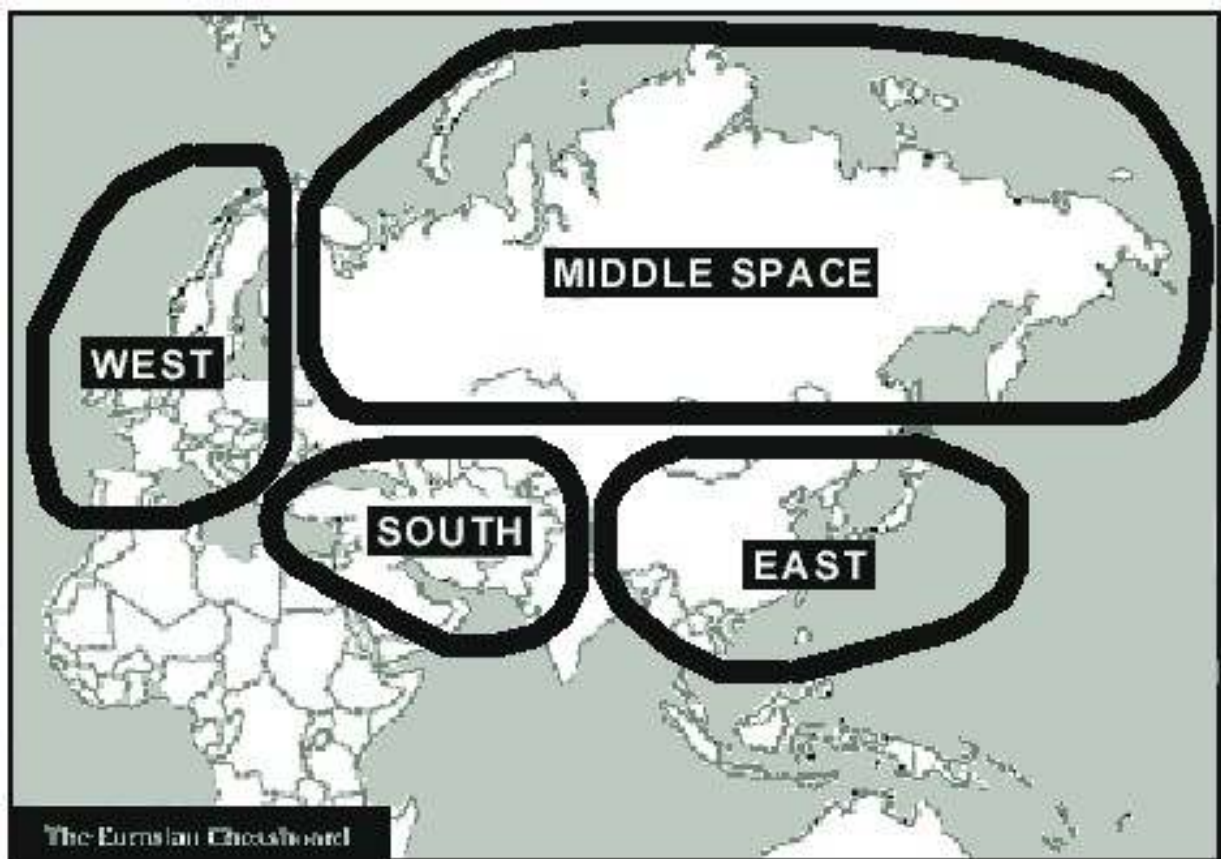

Source : Bibliotecapleyades.net

Depuis le divorce stratégique fracassant entre les États-Unis et le régime des mollahs et la fin de la guerre froide, Washington est revenu à une version plus classique, plus mackinderienne, de sa doctrine géopolitique, tout en continuant à regarder l'Iran à travers le prisme géopolitique (Kissinger). Qu'elle s'exprime à travers une politique de la " main tendue » (Clinton, Obama) ou qu'elle prenne le ton de la confrontation (Reagan, Bush, Trump), la politique iranienne des États-Unis obéit toujours aux mêmes fondamentaux : garder le contrôle du Rimland et éviter que les états qui le composent ne tombent définitivement dans l'orbite de Moscou (Cohen, 32; Brzeziński, 1997, 30-56). Dans cette optique et dans le contexte des bouleversements occasionnés par le Printemps arabe et le conflit irako-syrien, les géopoliticiens américains observent avec inquiétude l'influence grandissante de la Russie dans cette zone stratégique clé, son retour tonitruant sur l'échiquier moyen-oriental et les liens privilégiés noués avec la République islamique (Slykov, 44-49). Qu'il continue d'échapper ou non à la sphère d'influence occidentale, l'Iran demeure plus que jamais aux yeux des stratèges américains un outil dont dépend la réalisation de ses ambitions à l'échelle eurasiatique.

\section{Le point de vue iranien}

Sans surprise, la conception qu'a l'Iran de son rôle géopolitique contraste avec celui de « moyen » dans lequel les puissances régionales ou extrarégionales ont eu tendance à le cantonner. Pour les Iraniens, leur pays n'a vocation à être ni un corridor d'invasion, ni une "terre à prendre", ni un pion stratégique, ni un État tampon, ni un simple débouché économique. À leurs yeux, l'Iran possède sa propre finalité, celle d'une puissance souveraine poursuivant une politique étrangère affranchie des ingérences, pressions et menaces extérieures. Loin de n'être qu'une évidence logique, la perspective iranienne se construit d'abord, de manière dialectique, en opposition à la « 
fonction géopolitique » que lui ont assignée, tout au long de son histoire, les autres états (et leurs géopoliticiens). Dans une très large mesure, elle résulte aussi d'une vision du monde et d'une culture stratégique qui imprègnent profondément la psyché collective des Iraniens et de leurs dirigeants politiques.

\section{A. Sentiment d'exceptionnalisme} place légitime au sein du Panthéon des Grandes puissances. Cette aspiration à la grandeur et à la reconnaissance émane de la nostalgie de la grandeur passée et de la fierté qu'ont les Iraniens d'être les héritiers d'une grande civilisation. Loin d'être l'apanage des seuls nationalistes ou d'une élite intellectuelle, ce sentiment d'orgueil et d'exceptionnalisme est très largement partagé par les Iraniens de toutes obédiences et de toutes confessions.

C'est à l'aune des nombreux défis extérieurs auxquels l'Iran a fait face depuis vingt-cinq siècles et à travers sa capacité à relever ces périls incessants que s'est construite son identité stratégique et son refus de jouer un simple rôle de soutien dans le jeu international (Kinzer, 2008). Comme le rappelle S. Hunter, aucune des invasions et des ingérences extérieures n'est parvenu à altérer véritablement la personnalité ethnoculturelle de l'Iran ou à dissoudre ses aspirations ancestrales (Hunter). Tout au plus l'adversité a-t-elle exacerbé chez les Iraniens un fort complexe de souveraineté, un sentiment d'exceptionnalité et un désir profond de voir leur patrie retrouver "sa" place à la table des grandes puissances.

Cette éternelle prétention est une constante de l'Histoire qui, malgré les éclipses de la civilisation perse, s'incarne aussi bien dans le kémalisme des Pahlavi que dans le nationalisme d'un Mossadegh, dans l'approche offensive (Tahajomi) d'un Ahmadinejad que dans la stratégie de détente (Tashanojzedai) d'un Hassan Rouhani. Vingt ans après la révolution islamique, l'ayatollah Khatami rappelait devant les ruines de Pasargades que « les Perses furent un jour un peuple puissant et ingénieux » (IRNA). Si la nostalgie de la grandeur révolue continue aujourd'hui de guider les Iraniens, elle n'est pas exempte d'une profonde frustration: «Grand peuple à la culture plurimillénaire, notait Alexandre Adler, les Persans ont du mal à comprendre pourquoi ils sont tombés si bas et se sont souvent, par voie de conséquence, enthousiasmés pour des raccourcis saisissants vers le retour au pouvoir » (Adler, 3). Il n'en reste pas moins que les menaces subies tout au long de son histoire et les réflexes développés pour " préserver ses caractéristiques propres et les rendre offensives pour mieux les protéger » constituent l'une des tendances lourdes de l'identité stratégique de l'Iran et de sa façon de concevoir son « rôle » géopolitique (Réveillard).

\section{B. Syndrome de citadelle assiégée}

40 Le sentiment de supériorité coexiste avec un profond sentiment de fragilité géopolitique qui découle d'abord de l'histoire chaotique de l'Iran et de la suite incessante d'invasions et d'ingérence de toutes sortes qui l'ont ponctué - des conquêtes macédoniennes du IVe siècle av. J.-C. à l'opération Stuxnet découverte en 2010. Parmi elles, l'Opération Ajax d'aout 1953 planifiée par le MI6 et la CIA est l'un des «événements fondateurs de l'Iran moderne " dans la mesure où il a "foulé aux pieds le rêve d'indépendance nationale en infligeant à la mémoire nationale cette blessure encore aujourd'hui 
sensible» (Réveillard). Cette succession ininterrompue d'interférences étrangères a cultivé chez les Iraniens un syndrome de citadelle assiégée qui se traduit par la croyance quasi paranoïaque qu'à tout moment les puissances régionales et extrarégionales ourdissent des plans pour s'immiscer dans les affaires intérieures du pays, limiter sa souveraineté et mettre la main sur ses richesses hydrocarbures. Et Christophe Réveillard d'ajouter : "La méfiance à l'égard de ce monde par ailleurs objet de toutes les fascinations est la chose la mieux partagée en Iran » (Réveillard).

\section{Document 7 : Great Game cartoon from 1878}

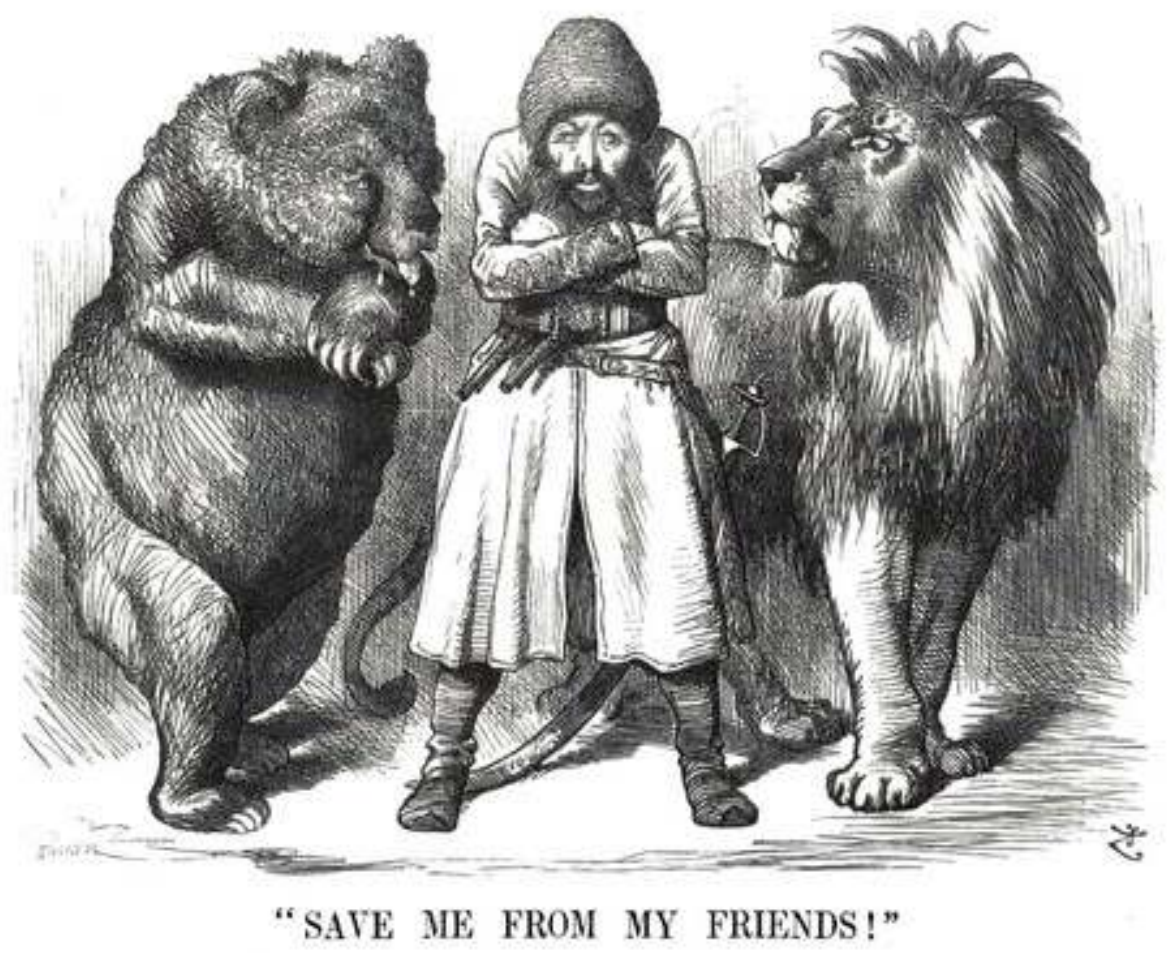

Source : Commons.wikimedia.org

41 Ce sentiment de vulnérabilité va de pair avec un 'complexe d'obsidionalité', à savoir l'impression d'être enclavés dans un environnement régional et international particulièrement hostile. Facette de la personnalité géopolitique de l'Iran que confirme François Thual : "L'Iran a toujours perçu sa position géopolitique sous le mode obsidional, dans l'appréhension constante des menaces que font peser sur lui les états ou les nations qui l'entourent » (Thual, 45). Frédéric Tellier souligne le poids de ce syndrome de citadelle assiégée sur la Weltanschauung des Iraniens : "Pèse ainsi sur les représentations psychologiques qui façonnent la diplomatie iranienne, une crainte pathologique de l'encerclement». C'est, par exemple, cette anxiété stratégique qu'expriment les dirigeants iraniens lorsqu'ils parlent de " cercles de feu " pour invoquer la présence d'une demi-douzaine de puissances nucléaires régionales ou extrarégionales à leurs frontières.

Ironiquement, le sentiment d'encerclement découle également de la capacité qu'a eue l'Iran de préserver son identité culturelle malgré les invasions et les influences étrangères. L'Iran n'a été hellénisé, arabisé ou turcisé qu'en surface et islamisé qu'à sa manière puisqu'il s'est débrouillé pour adapter le sunnisme à son propre caractère 
national. De même, il a subi l'impérialisme occidental tout en demeurant l'un des seuls pays du tiers-monde à ne pas avoir été formellement colonisé. Or, cette résilience identitaire comporte un prix stratégique : une profonde solitude culturelle et géopolitique. Seul pays persan et chiite dans un voisinage à majorité turcique et arabe, note S. Hunter, l'Iran ne peut compter, face aux menaces extérieures, sur aucune solidarité ethnoculturelle naturelle (Hunter). À toutes ces raisons qui sous-tendent le sentiment de vulnérabilité de l'Iran s'en ajoute une dernière : ce pays est à la fois trop grand et trop petit. Trop grand pour ne pas rentrer dans les calculs stratégiques des grandes puissances et pour ne pas attiser leur convoitise. Trop petit - et donc trop faible - pour inhiber leurs appétits.

\section{Deux impératifs et trois options}

De cette double volonté de survie et de puissance émergent deux impératifs stratégiques: protéger la forteresse iranienne en préservant son autonomie économique et son intégrité territoriale tout projetant hors de ses enceintes son influence à l'échelle régionale. Ces deux réflexes apparemment contradictoires ne le sont pas. Au sujet de ce dualisme stratégique, Tellier note : "si l'Iran brave la communauté internationale c'est qu'il sait que l'enjeu est la sanctuarisation de l'État-Nation iranien prélude à une nouvelle phase d'expansion idéologique " (Réveillard). La politique étrangère de l'Iran trouve toute sa cohérence dans la poursuite de cette double ligne stratégique qui transcende les clivages idéologiques et qui a été pratiquée par tous les régimes qui se sont succédé à la tête de l'Iran au cours des quatre derniers siècles.

Si les dirigeants iraniens savent où ils vont, il leur est en revanche plus difficile de déterminer avec quels partenaires s'associer pour y parvenir. Au cours de son histoire récente, l'Iran a recherché des alliés stratégiques dans chacun de ses voisinages géopolitiques - l'Occident, le Monde arabo-musulman, l'Eurasie - avec, dans chaque cas, des résultats contrastés et des défis spécifiques.

Faire alliance avec l'Occident présente a priori plusieurs avantages stratégiques pour l'Iran : à celui de développer des liens noués dès l'époque hellénique s'ajoutent ceux de travailler avec des puissances relativement lointaines lui permettant de s'affranchir du jeu régional et d'accélérer la réintégration dans le Gotha des grands états sans courir le risque d'une invasion directe ou d'un conflit ouvert. Mais, historiquement, le pari s'est avéré décevant pour les Iraniens. Les Occidentaux se sont imposés à l'Iran plus qu'ils n'ont été délibérément choisis. Surtout, ces puissances impériales centrales s'accommodent mal du désir d'indépendance et des ambitions géopolitiques des Iraniens. Contre l'Iran et ses intérêts, les pays européens ont souvent fini par s'entendre. C'est, entre autres, Napoléon qui abandonne le chah sur le radeau de Tilsit (1807), Londres qui s'entend avec Saint-Pétersbourg pour dominer le royaume Qadjar (1907), Hitler qui « offre » l'Iran à Molotov (1940), Staline et Churchill qui le partagent en zones d'influence (1941) ou encore Londres et Washington qui rognent sa souveraineté (1953-1979). Chacun de ces épisodes a été pour les dirigeants iraniens l'occasion d'apprendre à leurs dépens que le ménage avec l'Occident, ponctué de trahisons, comporte des bénéfices mais aussi des coûts considérables.

Voyant dans le monde musulman la promesse de pouvoir développer des liens plus équilibrés et moins risqués pour la souveraineté nationale avec des pays de la même taille que l'Iran, c'est vers cette option que se tournent les ayatollahs et les gardiens 
lorsqu'ils s'emparent du pouvoir en 1979. Mais, leurs efforts pour fédérer les états musulmans au sein d'un "gouvernement islamique global» (Pahlavi, 51) se sont heurtés à des obstacles géopolitiques significatifs. Le premier est de nature idéologique. Vue des sables du Hedjaz, l'ancienne Perse contre laquelle Mahomet avait unifié l'Arabie, reste une rivale - l' «ennemie proche " par opposition à l'« ennemi lointain » que constitue l'Occident. Éloignés par les dogmes et l'Histoire, les deux frères ennemis de l'Umma le sont également par les intérêts stratégiques. En raison de la logique de jeu à somme nulle qui caractérise le jeu moyen-oriental, tout gain engrangé par l'Iran sera systématiquement interprété comme une perte sèche par les puissances arabes et sera donc automatiquement bloqué par ces dernières (Pahlavi, 45-52). À elle seule, cette logique explique l'aggravation sensible des lignes de fracture politiques, ethniques et religieuses observées depuis l'accord nucléaire 2015. De sorte que si le Moyen-Orient demeure pour l'Iran une voie à examiner, la région constitue également un cul-de-sac stratégique pour ses ambitions internationales.

Document 8 : L'Organisation de coopération de Shanghaï

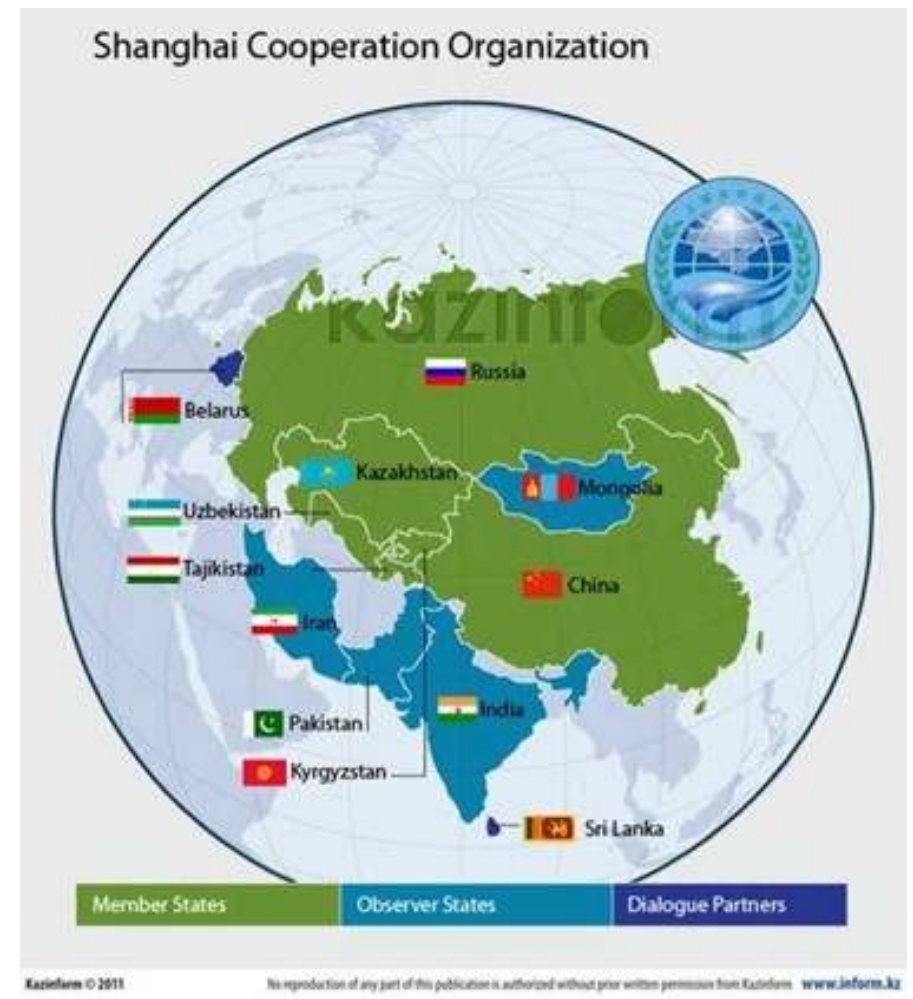

Source : Thetruthsource.org

Une " troisième voie " consiste pour l'Iran à développer un axe de coopération eurasiatique avec la Russie, la Chine ou l'Inde. Des pays qui, en plus de promouvoir un ordre mondial plus multipolaire, partagent avec l'Iran une posture farouchement westphalienne ne souffrant aucune remise en cause des principes de souveraineté et de non-ingérence. Du Bosphore à Formose, mollahs et gardiens se démènent depuis plusieurs années pour façonner autour de l'Iran un bouclier d'amitié. En témoignent la candidature iranienne à l'Organisation de coopération de Shanghai (OCS) et les liens stratégiques noués avec Pékin et Moscou. Reste à savoir si cette Ostpolitik pourra offrir le socle géopolitique dont l'Iran a besoin. Certes, observent les experts, « avec l'Iran 
dans la poche ", ces puissances eurasiatiques disposent d'une entrée au Moyen-Orient mais il est permis de douter qu'elles partagent équitablement leurs bénéfices avec leur modeste partenaire iranien (Kantham). En rejoignant cette "périphérie réaliste " opposée au «noyau postmoderne» des pays occidentaux (Goldgeier et McFaul, 1-26), l'Iran court le risque d'être relégué a un rôle de sous-fifre dans une entreprise dirigée par des poids-lourds qui lui feront d'autant plus d'ombre que, contrairement aux puissances extrarégionales, elles opèrent dans son voisinage immédiat.

\section{Conclusion}

La géopolitique ne se limite pas à l'étude des déterminants physiques, géographiques et stratégiques qui influencent les pays. Elle est également un prisme qui forme et déforme l'interprétation que font les états de leur place et de celles des autres dans le jeu international. À travers ce prisme, un pays comme l'Iran est vu, construit, reconstruit et confiné dans une série de fonctions qui définissent et pérennisent sa trajectoire et ses interactions autant que le contexte géographique dans lequel il opère. À quelques nuances près, le rôle qu'assignent les autres états à l'Iran se résume invariablement à celui d'un outil, d'un instrument, pouvant et devant être mis au service de leurs propres intérêts. Vu par ses voisins eurasiatiques, l'Iran est un passage sur un axe de conquête, un corridor d'invasion, une étape sur une voie commerciale, un verrou stratégique vers le Nord ou un accès vers les mers chaudes du Sud. Vu d'outremer, de l'Occident lointain, l'Iran se voit plutôt attribué une fonction de pion stratégique, d'État tampon, de source d'énergie ou de débouché économique.

Le fait notable est qu'aucune des fonctions qui lui sont assignées de l'extérieur ne correspond au rôle que l'Iran ambitionne lui-même de jouer sur la scène internationale : celui d'un État fort et autonome désireux de poursuivre une politique de grande puissance affranchie des diktats de la communauté internationale. Or, bien que l'Iran ne manque pas d'atouts pour réaliser ses ambitions, ses efforts buttent sur des obstacles majeurs dont le moindre n'est pas son confinement systématique à une fonction de pion stratégique. À la difficulté tangible de la situation centrale et délicate de l'Iran dans une zone sensible et sous tension quasi-permanente s'ajoute en effet le défi, plus intangible, de la manière qu'ont les autres états de percevoir sa place et sa fonction dans le jeu international.

Paradoxalement, en opposant à cette vision d'un Iran " instrument marginal » des autres puissances celle d'une Perse "centre du monde ", les Iraniens ne réussissent qu'à faire apparaître davantage l'isolement de leur pays et la limitation de sa marge de manœuvre. Tout, de sa situation d'encerclement à son "complexe d'obsidionalité ", contribue à faire de l'Iran une " citadelle assiégée " contrainte, pour assurer sa pérennité et assouvir son désir de rayonnement, à ne s'en remettre qu'à ses propres moyens. Compte tenu de cette équation complexe, note S. Hunter, «l'Iran a besoin d'une approche essentiellement nationaliste, autonome, pragmatique et nonidéologique de la politique étrangère ». Cette approche froide et calculée, qui implique un vrai Thermidor, doit consister à éviter d'être entraîné dans les conflits et les intérêts des autres puissances ou de s'impliquer aveuglément et exclusivement dans un axe d'alliance plutôt qu'un autre (Hunter). Privé d'une alliance stratégique et à défaut de posséder les moyens de s'affranchir du carcan que lui impose son statut géopolitique ou de transcender le rôle dans lequel l'enferment les autres états, l'Iran doit combiner les 
atouts dont il dispose de manière à contourner ses faiblesses relatives dans les domaines économique, technologique et militaire.

51 Contrainte à compter sur elle-même et à faire avec les moyens du bord, la vielle Perse se tourne, comme souvent au cours de son histoire, vers une approche asymétrique qui consiste à éviter la confrontation directe avec les adversaires régionaux et extrarégionaux, à agir là où elle jouit d'avantages comparatifs (notamment idéologiques et paramilitaires) tout en concentrant ses efforts sur les deux impératifs permanents qui ont toujours guidé sa politique étrangère : protéger la forteresse iranienne et assurer son rayonnement régional de la manière la plus prudente et la plus pragmatique possible. En attendant de combler ses faiblesses, l'outsider iranien continuera de pratiquer cette approche développée par les Ismaéliens et les Parthes. Esquivant le combat frontal et agissant là où on ne l'attend pas, l'Iran s'efforcera, pour reprendre le mot de Kissinger, de " gagner en ne perdant pas ». Cette stratégie s'est déjà révélée payante, et il est à parier que Téhéran continuera à jouer de la sorte tant que cela lui permettra d'engranger des gains et d'accélérer sa remontée vers l'olympe des grands états (Dorsey).

\section{BIBLIOGRAPHIE}

Adib-Moghaddam Arshin, Psycho-nationalism: Global Thought, Iranian Imaginations (The Global Middle East), Cambridge, Cambridge University Press, 2018.

Adler Alexandre, « Pourquoi il ne faut pas désespérer de l’Iran », Le Figaro, 14 octobre 2007.

Al-Zahed Saud, "Hatred of Arabs deeply rooted in Persians", Al Arabiya News, octobre 2011.

Asharq Al-Awsat, "The Middle East between neo-Ottomans and neo Safavids," TrackPersia, octobre 2016.

Mostafa Azarian, «La Russie va-t-elle créer des bases militaires dans le golfe Persique ? », Press TV, 13 aout 2017.

Gueynard Bertrand, « Near East ou Middle East: histoire d'une terminologie », Outre-Terre, vol. 13, no. 4, 2005.

Brzeziński Zbigniew, Entretien, Nouvel Observateur, 15-21 janvier 1998.

Chaliand Gérard, Préambule, Atlas stratégique, Paris, Fayard, 1993.

Kantham Chris, "Iran Protests through the Prism of Geopolitics", Sott.net, janvier 2018.

Réveillard Christophe, «L'Iran géopolitique », Académie de Géopolitique de Paris, Avril 2006.

Clawson Patrick, Eternal Iran, New York, Palgrave Macmillan, 2005.

Bosworth Clifford E., “Ajam,” Encyclopædia Iranica, 1985.

Dugin Aleksandr, Osnovy geopolitiki. Geopoliticheskoe budushchee Rossii (Fondamentaux de géopolitique: Fondamentaux de géopolitique : l'avenir géopolitique de la Russie), Moscou, Artogeia, 1997. 
Tulin Daloglu, "Davutoglu Invokes Ottomanism as New Mideast Order," Al-Monitor, Mars 2013 Mohammad-Reza Djalili, Géopolitique de l'Iran, Bruxelles, Complexe, 2005.

Blanchard Jean-Marc, "The Geopolitics of China's Maritime Silk Road Initiative," Geopolitics, vol. $22, n^{\circ} 2,2017$.

Burman Edward, China and Iran: Parallel History, Future Threat?, New York, The History Press, 2009.

Suleyman Elik, Iran-Turkey Relations, 1979-2011: Conceptualising the Dynamics of Politics, Religion and Security in Middle-Power States, New York, Routledge, 2013.

Erdbrink Thomas, "For China's Global Ambitions, 'Iran is at the Center of Everything,," New York Times, juillet 2017.

Fatemeh Shayan, Security in the Persian Gulf Region, New York, Springer, 2017.

Geranmayeh Ellie et Kadri Liik, "The new power couple: Russia and Iran in the Middle

East," European Council on Foreign relations, 2016.

Thual, François, Géopolitique du Chiisme, Arléa, 2002.

Frye. Richard Nelson et al., Cambridge History of Iran, vol 4, Londres, 1975.

Gaume Jean-Joseph, Le Testament de Pierre-Le-Grand ou la Clef de L'avenir, Paris, Saint-Rémi, 1876.

Scott W. Harold et Nader Alireza, China and Iran: Economic, Political, and Military Relations, RAND corp., 2012.

Heller Michel, «Le testament de Pierre le Grand », Histoire de la Russie et de son empire, Paris, Éditions Perrin-Tempus, 2015.

Hunter Shireen, “Iran's Geopolitical Predicament and Its Consequences”, Loble Log, Mars 2017.

Ilminskaya M.F. “The Persian Gulf Region as a Field of China's Geopolitical Interests," Vestnik RUDN, $\mathrm{N}^{\circ} 1,2015$.

Goldgeier James et McFaul Michael, «The liberal core and the realist periphery», Perspectives on European Politics and Society, volume 2, no1, 2001.

Dorsey James M., “Iran's Surprisingly Strong Geopolitical Hand,” The Globalist, March 2017.

Jirinovski Vladimir, послЕДний БРосок НА юг [The Last Break Southward], Moscou, Foliant, 1995.

Tancrède Josseran, La nouvelle puissance turque, Paris, Ellipses, 2010.

Kaplan Robert D., The Revenge of Geography: What the Map Tells Us About Coming Conflicts and the Battle against Fate, New York, Random House, 2013.

Kapuscinski Ryszard, Shah of Shahs, London, Houghton Mifflin Harcourt, 1985.

Kinzer Stephen, “Inside Iran's Fury,” Smithsonian magazine, Octobre 2008.

Kinzer Stephen, Reset: Iran, Turkey, and America's Future, New York, Macmillan, 2010.

Klaus Dodds (ed.), Geopolitics, Londres, Sage, 2010.

Mackinder H.J., "The Geographical Pivot of History,” The Geographical Journal, vol. 24, no. 4, 1904. 
Mousavian Seyed Hossein, “The GCC's three options for dealing with Iran," Al-Monitor, Mars 2017.

Pahlavi Pierre et Pahlavi Christian, Le Marécage des Ayatollahs : Une histoire de la révolution iranienne de 1979, Paris, Perrin-Tempus, 2017.

Pahlavi Pierre. « Quel rôle géopolitique pour l'Iran ? ", Géopolitique de l'Iran, Les Grands Dossiers de Diplomatie n³7, Diplomatie, 2017.

Shahandeh Behzad et Maaike Warnaar, "Prospects for Change in GCC-Iran Relations?", in M. Warnaar et al., Iran's Relations with the Arab States of the Gulf: Common Interests over Historic Rivalry, Berlin, Gerlach Press, 2016.

Shariatinia M., "Iranophobia: Roots and Repercussions," Foreign Relations, vol. 2, no.1, 2010.

Shlapentokh Dmitry, "Russian Elite Image of Iran: From the Late Soviet Era to the Present," Strategic Studies Institute, U.S. Army War College, 2009.

Sloan G.R., Geopolitics in United States Strategic Policy, 1890-1987, New York, St. Martin's Press, 1988.

Slykov Pavel, "Russian Foreign Policy in the Eastern Mediterranean since 1991," in S. Litsas et A. Tziampiris eds., Multipolarity, Politics and Power, Piraeus, Ashgate, 2014.

Spykman N. J., The Geography of the Peace, New York, Harcourt, Brace, 1944.

Tiersky Ronald, “The Geopolitics of Respect: U.S., China, Iran and Russia”, HuffingtonPost, Sept. 2015.

Trenin Dmitri, "Russia and Iran: Historic Mistrust and Contemporary Partnership," Carnegie Moscow Center, 2016.

Villemarest Pierre F. de, « Lettre d'information », Centre européen d'information, Bulletin du 16 octobre 1980.

Wright Robin, “Iran's New Alliance with China Could Cost U.S. Leverage," Washington Post, novembre 2004.

Zarrinkoub Abdolhosein, Two Centuries of Silence, Londres, Mazda Publications, 2017.

« President Khatami back to Tehran after visiting Persepolis », IRNA, 19 Janvier 2001.

« Maqam mo'azzam-e rahabari zaban-e Amrika va Abargodrat-ha ra zaban-e tahdid va er'ab danestand », IRNA, 28 février 2008.

« Iranian official predicts establishment of Iran-led international Islamic government this century », Middle East Monitor, 29 novembre 2016.

\section{NOTES}

1. En persan : Arvand Rud.

2. La signification littérale du toponyme «Irān ».

3. Ironiquement, en leur conférant une légitimité scientifique, les géopoliticiens contribuent également à la pérennisation des scénarios et des distributions de rôles géopolitiques. 


\section{RÉSUMÉS}

Pays charnière, verrou stratégique, carrefour de communication, nœud de pèlerinage et d'échange commerciaux, l'Iran possède, à plus de titres, une réputation de pivot géopolitique. Pour autant, sa trajectoire n'est pas uniquement déterminée par sa place centrale sur la mappemonde. En marge de la géopolitique classique qui se limite à l'impact de la géographie physique, une voie alternative consiste à examiner la manière dont cette réalité permanente et objective est prolongée, reconstruite et réinterprétée historiquement sous la forme d'un prisme subjectif qui détermine à son tour la perception que les états ont les uns des autres et de leur rôle respectif dans le jeu international. À travers ce prisme, un pays comme l'Iran est vu, construit, reconstruit et confiné dans une série de «fonctions géopolitiques » qui définissaient pérennisent ses interactions autant que le contexte géographique tangible dans lequel il évolue. À quelques nuances près, le rôle qu'assignent les autres états à l'Iran se résume invariablement à celui d'un moyen stratégique pouvant et devant être mis au service de leurs propres intérêts. Vu par ses voisins eurasiatiques, l'Iran est un passage sur un axe de conquête, un corridor d'invasion, une étape sur une voie commerciale, un verrou stratégique vers le Nord ou un accès vers les mers chaudes du Sud. Vu d'outremer, de l'Occident lointain, l'Iran se voit plutôt attribué une fonction de pion stratégique, d'État tampon ou de débouché économique. Le fait est qu'aucune des fonctions qui lui sont assignées de l'extérieur ne correspond au rôle que l'Iran ambitionne luimême de jouer sur la scène internationale : celui d'un État autonome et désireux de poursuivre une politique de grande puissance souveraine et affranchie des diktats de la communauté internationale. L'objectif de cet article est de démontrer que, en plus d'être influencés par les paramètres d'ordre géostratégique, l'Iran et son destin sont très largement façonnés par la contradiction profonde qui existe entre les "fonctions " géopolitiques que lui attribuent les autres états, régionaux et extrarégionaux, et le rôle que ce pays estime devoir être le sien sur l'échiquier moyen-oriental et international. La mise en contraste de ces visions géopolitiques souvent divergentes - permet de faire apparaitre la dimension éminemment complexe et conflictuelle de la relation entre l'Iran et ses partenaires internationaux ainsi que le nombre limité des options stratégiques qui s'offre à ce pays dans la poursuite de ses ambitions et objectifs stratégiques.

Described as a linchpin, a strategic lock, a communication crossroads, a pilgrimage node or a trade hub, Iran has, in many ways, a reputation of geopolitical pivot. However, it would be a mistake to overstate the importance of its central position on the world map. Beyond classical geopolitics, which focuses on the impact of physical geography, an alternative way is to examine how this permanent and objective reality is prolonged, reconstructed and reinterpreted historically in the form of a subjective prism that determines in turn the perception that states have of each other and of their respective roles on the international stage. Through this prism, a country like Iran is seen, constructed, reconstructed and confined to a series of functions that define and perpetuate its interactions as much as the tangible geographic context within which it operates. With a few slight variations, the role that other states assign to Iran invariably amounts to that of a mere instrument that can and must be put at the service of their own interests. Seen by its Eurasian neighbors, Iran is viewed as a passage on an axis of conquest, an invasion corridor, a counter on a commercial route, a strategic bolt to the North or a bridge to the warm southern seas. Seen from far-off West, Iran is rather attributed a function of a strategic pawn, a buffer state, an influence platform or an economic outlet. A key fact is that none of the functions assigned to it from the outside corresponds to the role that Iran itself aspires to play on the international scene: that of a sovereign State willing to pursue a policy of great power 
emancipated from the dictates of the international community. The objective of this article is to demonstrate that, in addition to being influenced by tangible geostrategic parameters, Iran and its behavior are very largely shaped by the profound contradictions that exist between the geopolitical "functions" attributed to it by other states, regional or extra-regional, and the role that this country feels should be its own on the Middle Eastern and international chessboard. Contrasting these geopolitical visions - often divergent - makes it possible to reveal the profoundly complex and conflictual dimension of the relationship between Iran and its international partners, as well as the limited number of strategic options available to this country to pursue its ambitions and strategic objectives.

INDEX

Mots-clés : Iran, Moyen-Orient, géopolitique, stratégie, Eurasie, États-Unis, Russie, Chine, mondes arabe et musulman

Keywords : Iran, Middle East, geopolitics, strategy, Eurasia, United States, Russia, China, Arab and Muslim Worlds

\section{AUTEUR}

\section{PIERRE PAHLAVI}

Professeur au Collège des forces canadiennes (Toronto), directeur adjoint du Département des études de la défense et directeur du département de la sécurité internationale 